\title{
Violation of bilocality in quantum networks
}

\section{Cyril Branciard}

Univ. Grenoble Alpes, CNRS, Grenoble INP, Institut Néel, 38000 Grenoble, France

\begin{abstract}
Bell nonlocality-that observations cannot be explained in terms of local influences-is one of the most remarkable features of quantum theory, and has found interesting applications for quantum information processing. A variant of it is now observed in basic quantum networks involving independent sources, with a similar potential for applications.
\end{abstract}

Despite the great role he played in the development of quantum theory, Albert Einstein was notoriously unsatisfied with the theory. In 1935, together with his colleagues Boris Podolsky and Nathan Rosen[1], he described a situation predicted by quantum theory, where a pair of particles could be in such a state-a so-called entangled state-that performing a measurement on one particle seemed to instantaneously affect the other particle. According to quantum theory, the very strong correlations between these entangled particles would survive, whatever the distance between them. Such a "spooky action at a distance" was clearly unacceptable for the father of special and general relativity. For him, the only possible explanation should be that the particles carry some physical property, which would locally determine measurement results: the correlations would be established at the source, where the pair of particles was created. As quantum theory did not describe such a property, Einstein concluded that the theory was incomplete, and would thus need to be completed so as to include additional "local variables".

In 1964 however, John Bell showed ${ }^{2]}$ that quantum theory could not be completed as Einstein hoped for. Indeed, the correlations obtained in any model with local variables are limited by some specific bounds, known as Bell inequalities-while quantum correlations were predicted to violate these inequalities. And indeed in the last four decades experimental groups obtained Bell inequality violations in more and more advanced/convincing experiments, finally dealing a few years ago ${ }^{[3]}$ a fatal blow to Einstein's intuition about the world: the observed correlations between entangled particles cannot be explained by local variables (Fig. 1a), and we have to accept that our world is (in this sense) "nonlocal".

The situation becomes even more puzzling when considering the situation of Fig. 1b, involving two independent quantum sources $\left(S_{1}, S_{2}\right)$ and three observers (call them Alice, Bob and Charlie). Here $S_{1}$ and $S_{2}$ send pairs of particles to Alice and Bob, and to Bob and Charlie, respectively. For a given joint measurement preformed by Bob, the particles of Alice and Charlie may end up entangled-even though they never interacted directly, and have no common past: the entanglement between Alice and Bob, and between Bob and Charlie, is "swapped" to be shared by Alice and Charlie ${ }^{[4]}$.

These new entangled particles could then show nonlocality. However, recalling the intuition about the local variables originating from the sources, it would be natural in this situation to test models with two independent local variables originating from two 
independent sources, with one variable being sent to Alice and Bob, and one variable sent to Bob and Charlie. This further restricted class of models is called "bilocal". Similar to Bell inequalities, "bilocal inequalities" can be constructed, that constrain correlations obtained from bilocal models ${ }^{[5]}$. Again quantum theory predicts violations of bilocal inequalities, as verified experimentally by Sun et al., who report their results in Nature Photonics ${ }^{[6]}$ : quantum correlations in the setup of Fig. $1 \mathrm{~b}$ cannot be explained by any bilocal model.

\section{Loopholes}

In order to be conclusive, tests of Bell inequalities, or of bilocal inequalities, must meet quite demanding requirements-otherwise they are easily prone to loopholes, so that what looks like a violation obtained experimentally could in fact be explained by some (possibly quite far-fetched) local variable model.

The most significant loopholes for Bell inequalities are (i) the "locality loophole", which arises when the relevant events on Alice and Bob's sides (Fig. 1a)-from them choosing some measurement to perform on their respective particle, to realising the measurement and getting a result-are not space-like separated, as subluminal communication could then perfectly explain the correlations; (ii) the "measurement independence loophole", which arises if measurements performed on each side are not "freely chosen", but could depend e.g. on the state sent by the source of entangled particles; and (iii) the "detection loophole", which arises when not all particles are detected, as in this case some custom-made local model and naïve postselection could fake a Bell inequality violation. It took decades for Bell tests, mostly realised with photon pairs, to close these loopholes one after the other, and finally close them altogether in the same tests-thus demonstrating what are now generally considered to be "(significant-)loophole-free" Bell inequality violations ${ }^{[3]}$.

Bilocality tests are also prone to similar loopholes. There is however another crucial potential loophole to consider: testing a bilocal model only makes sense if the quantum sources used in the experiment are truly independent. This "source independence loophole" is however tricky: it is in fact impossible to guarantee that two sources are fully independent, as they always share a common past, and could have been correlated at the birth of the universe; all one can do is to make sufficiently convincing that the sources are independent, to make bilocal models reasonable enough to test.

Previous tests of bilocal inequalities used Spontaneous Parametric Down-Conversion sources, with two separate nonlinear crystals (where the photon pairs were created) pumped by the same laser ${ }^{[7]}$ in one of these experiments the phase of the pump laser was randomised between the two crystals, to further justify the source independence assumption. The experiment reported in this issue by Sun et al.[6] further improves on the source independence justification, by using two independent lasers pumping independent SPDC crystals. Sun et al. furthermore synchronised all events carefully enough to make sure the locality and measurement independence loophole are closed (with measurement settings chosen by quantum random number generators). There would just remain the detection loophole to close (which requires very high singlephoton detection efficiencies), in order to make their bilocal test "(significant-)loopholefree". 
It should be noted, however, that some of the recent "loophole-free Bell tests"[3] actually also used an "entanglement swapping" scheme as in Fig. 1b, with independent quantum sources. As these ruled out standard local variable models, they also a fortiori ruled out bilocal models (which make strictly stronger assumptions than local ones)-and could thus be also seen as "(significant-)loophole-free" tests of bilocality.

The novelty of the new test by Sun et al., compared to these previous Bell tests, is thus not so much the mere fact that it rules out bilocal models (up to the detection loophole). It is that it tested a bilocal models directly (through the violation of a bilocal inequality). In the future such bilocal tests may become quite relevant, in particular in the intermediate situation where local variable models can reproduce the observed correlations, but bilocal models cannot.

\section{Towards applications and generalisations}

It was realised in the last couple of decades that quantum nonlocality could have very interesting, practical applications in quantum information science: Bell inequality violations can e.g. used to guarantee the security of quantum key distribution, in a device-independent manner-i.e., without needing to trust the functioning of one's physical devices ${ }^{[8]}$.

Of course these applications break down when nonlocality cannot be observed. However, some might be rescued if they could be based on non-bilocality instead, in case the latter can still be demonstrated. It is indeed natural to expect non-bilocality to also find nice applications for quantum information processing in the setup of Fig. $1 \mathrm{~b}-$ indeed recent works started exploring this possibility ${ }^{[9]}$.

And beyond the rudimentary scenario of Fig. 1b, one may also explore non-locality in future, more complex networks. If those are constructed with independent quantum sources, as illustrated in Fig. 1c, then the bilocality assumption could naturally be generalised to " $N$-locality", for $N$ independent sources-i.e., one may test quantum correlations against models with networks of local variables with the same topology as the quantum network of interest. Applications are certainly to be found, that exploit the network structure. The experimental demonstration of Sun et al. should motivate further investigations along such directions, which will become more and more relevant with the development of quantum technologies, and of more and more sophisticated quantum networks.

\section{$\underline{\text { References }}$}

[1] Einstein, A., Podolsky, B. \& Rosen, N., Phys. Rev. 47, 777 (1935).

[2] Bell, J. S., Physics 1, 195 (1964).

[3] Hensen, B. et al., Nature 526, 682 (2015); Giustina, M. et al., Phys. Rev. Lett. 115, 250401 (2015); Shalm, L. K. et al., Phys. Rev. Lett. 115, 250402 (2015); Rosenfeld, W. et al., Phys. Rev. Lett. 119, 010402 (2017).

[4] Żukowski, M. et al., Phys. Rev. Lett. 71, 4287 (1993).

[5] Branciard, C., Gisin, N. \& Pironio, S., Phys. Rev. Lett. 104, 170401 (2010);

Branciard, C. et al., Phys. Rev. A 85, 032119 (2012).

[6] Sun, Q.-C. et al., Nat. Photon. 13, 687-691 (2019). 
[7] Carvacho, G. et al., Nat. Commun. 8, 14775 (2017); Saunders, D. J. et al., Sci. Adv. 3, e1602743 (2017).

[8] Brunner, N. et al., Rev. Mod. Phys. 86, 419 (2014).

[9] Ciarán M. Lee, C. M. \& Hoban, M. J., Phys. Rev. Lett. 120, 020504 (2018).

(a)

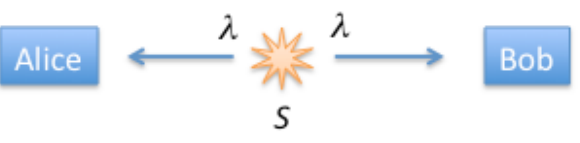

(b)

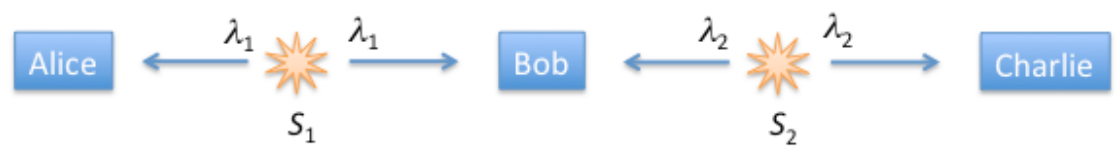

(c)

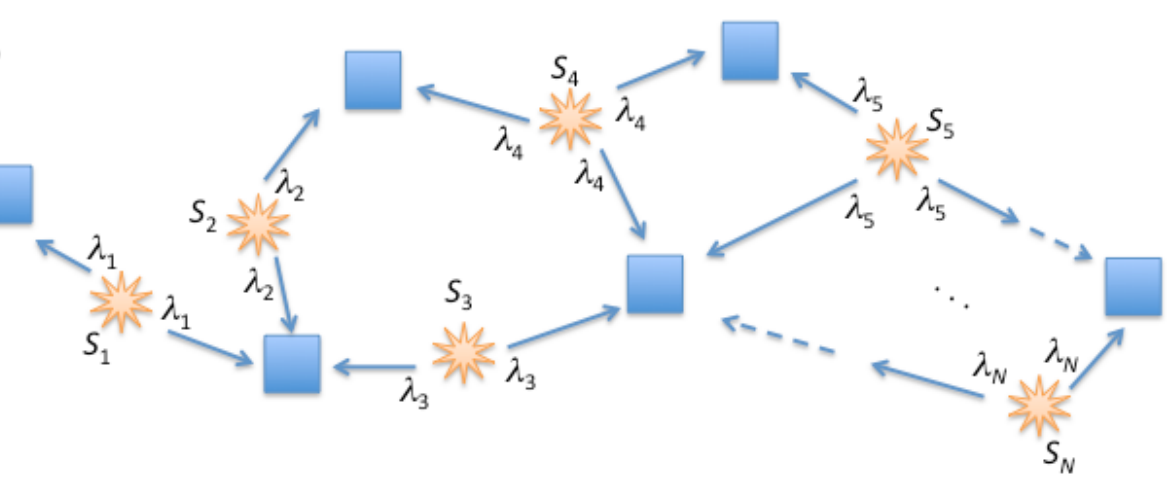

Figure 1. (a) Einstein's intuition was that measurement results on a pair of entangled particles should be determined locally, by some physical property—or "local variable" $(\lambda)$-established at the source of the particles. Bell proved him wrong: no local variable model can reproduce quantum correlations. (b) In a quantum experiment with independent sources, one may compare the observed correlations to those obtained from models with independent local variables $\left(\lambda_{1}, \lambda_{2}\right)$ originating from independent sources-so-called "bilocal models". Again quantum correlations are non-bilocal, as experimentally verified in this issue of Nature Photonics ${ }^{[6]}$. (c) Correlations established in future, more complex quantum networks involving $N$ independent sources could be tested against " $N$-local" models that assume the same network topology. Non- $N$-locality could find applications for quantum information processing ${ }^{[9]}$.

Published version:

Cyril Branciard,

Violation of bilocality in quantum networks,

Nature Photonics 13, 662-663 (2019).

https://doi.org/10.1038/s41566-019-0522-3 